\title{
High Dose Chemotherapy and Autologous Stem Cell Transplantation in Enteropathy-Associated and other Aggressive T- Cell Lymphomas: A UK NCRI/Cancer Research UK Phase II Study
}

Elizabeth H Phillips (1), Michelle M Lannon (2), Andre Lopes (1), Humra Chadwick (1), Gail Jones

(2), Michal Sieniawski (3), Andrew Davies (4), Katrina Wood (5), Laura Clifton-Hadley (1), Paul Smith (1), Anthony Lawrie (1), Nick Chadwick (1), Anne L Lennard (2)

1. Cancer Research UK and University College London Cancer Trials Centre, UK

2. Department of Haematology, Freeman Hospital, Newcastle Upon Tyne, UK

3. South Tyneside District Hospital, South Shields, UK

4. Cancer Research UK Centre, Cancer Sciences Unit, University of Southampton, Faculty of Medicine, Southampton, UK

5. Department of Cellular Pathology, Royal Victoria Infirmary, Newcastle Upon Tyne, UK

Correspondence to: $\underline{\text { b.phillips@ucl.ac.uk }}$ 
Outcomes for patients with peripheral T-cell lymphoma (PTCL) are generally poor with 35-40\% 5-year survival using CHOP-like chemotherapy. Addition of other agents to CHOP has not consistently improved outcomes and can cause excess toxicity ${ }^{1,2}$; new approaches are urgently needed. Favourable outcomes have been achieved with autologous stem cell transplant consolidation (ASCT) in some settings with long term survival rates exceeding $50 \%$, particularly for those transplanted in first complete remission ${ }^{3}$.

EATL is a rare form of T-cell lymphoma with an incidence of $0.1 / 100,000$ per year in Western Europe ${ }^{4}$. EATL poses a number of diagnostic and therapeutic challenges, as patients frequently present with poor performance status, malnutrition and acute abdominal complications. There is no established standard of care and outcomes are inferior to other T-cell lymphomas with 5-year failure free survival of $<5 \%{ }^{5-7}$. We have previously reported the use of intensive chemotherapy followed by ASCT in a retrospective population-based study of patients with EATL ${ }^{4}$. These favourable results encouraged us to prospectively assess the feasibility of this approach in a multi-centre setting for aggressive T-cell lymphoma.

Patients with histologically confirmed, previously untreated EATL aged $\geq 18$ years were eligible for entry into this single arm phase 2 trial. In light of emergent data on ASCT in PTCL and to increase recruitment, the protocol was amended to allow inclusion of patients with PTCL, ALK-negative anaplastic large cell lymphoma (ALCL) and extranodal NK/T-cell lymphoma (ENKTL). Performance status (PS) was permissive but patients had to be deemed able to tolerate the intensive treatment regimen by the treating clinician. Exclusion criteria included severe cardiac, renal or liver dysfunction. Disease was staged using Lugano (EATL patients) or Ann Arbor (all other patients) staging systems. Central histopathology review was undertaken in Newcastle upon Tyne Hospitals NHS Foundation Trust. Written informed consent was obtained prior to study entry. 
Patients received 1 cycle of CHOP chemotherapy (cyclophosphamide, doxorubicin, vincristine and prednisolone), followed by 3 cycles of IVE (ifosfamide $1500 \mathrm{mg} / \mathrm{m}^{2}$ bd days $1-3$, etoposide $200 \mathrm{mg} / \mathrm{m}^{2}$ days $1-3$ and epirubicin $50 \mathrm{mg} / \mathrm{m}^{2}$ day 1 ), with intermediate dose methotrexate (MTX, $1.5 \mathrm{~g} / \mathrm{m}^{2}$ ) administered on day 22 of each 28 day cycle (see Figure 1$)^{4}$. Peripheral blood stem cells were harvested after either 2 or 3 courses of IVE. Patients achieving CR after IVE-MTX, received high dose therapy with BEAM (carmustine, etoposide, cytarabine and melphalan) followed by ASCT ${ }^{4}$. Patients achieving partial response (PR) could also receive ASCT on trial at the investigator's discretion.

The primary endpoint was 1-year overall survival (OS) for EATL patients. With $80 \%$ power and $5 \% 2$ sided alpha, 60 patients would be required to detect an improvement in 1-year OS from 31\% to 54\% in EATL. Secondary endpoints included toxicity and regimen feasibility across multiple sites. Response was assessed at the end of IVE-MTX induction by contrast-enhanced computed tomography (CT) and/or by endoscopy and biopsy, according to standard criteria ${ }^{8}$.

Twenty-one patients were enrolled at 10 UK sites between September 2009 and July 2014; target recruitment was not reached. Clinical characteristics are detailed in Table 1. Median age was 55 years (31-71 years). Six patients (29\%) required laparotomy at diagnosis; all patients had measurable disease at study entry.

All patients received 1 cycle of CHOP chemotherapy. Eighteen patients (86\%) completed 3 cycles of IVE and 15 patients (71\%) completed 3 cycles of methotrexate. Grade 3-5 toxicity is detailed in Table 2. One patient (5\%) discontinued trial therapy due to grade 3 encephalopathy with ifosfamide. Four patients (19\%) required dose reductions for at least 1 cycle of IVE chemotherapy, mostly due to haematological toxicity. There were 2 fatal events during induction therapy: 1 neutropaenic sepsis, and 1 sudden death deemed due to pre-existing epilepsy +/- type 1 diabetes, which occurred 16 days 
after completing the final IVAC cycle with no preceding symptoms to suggest ifosfamide encephalopathy. This was not felt to be treatment-related.

Following completion of IVE-MTX induction therapy, 10 patients (48\%) achieved CR and 5 (24\%) achieved PR, with an overall response rate of $71 \%$ for the whole cohort. Three patients (14\%) had progressive disease (PD) and $3(14 \%)$ were not assessable due to death or study withdrawal. Similar response rates were achieved in the EATL cohort, with CR in 6 patients (55\%), PD in 2 (18\%) and not assessable in $3(27 \%)$.

Of the 15 patients that responded to IVE-MTX, 2 (13\%) failed to mobilise sufficient stem cells, precluding planned autograft. In total, 13 patients (62\%) proceeded to ASCT, including 5 of 11 (45\%) EATL patients. Five PTCL patients in PR after induction chemotherapy continued to ASCT at the clinicians' discretion, of which 2 (40\%) subsequently achieved CR.

Median follow-up was 27 months. Survival curves are shown in Figure 1. One-year OS and PFS for the whole cohort were $62 \%$ (95\% confidence interval (CI): $38-79)$ and $48 \%$ (95\% Cl: $26-67)$, respectively. Median PFS was 12 months and median OS has not been reached. For EATL patients, 1-year OS and PFS were both 45\% (95\% Cl: 17 - 71). Of the 5 EATL patients receiving ASCT, only 1 has relapsed. Two of 3 patients with presumed type 2 EATL (monomorphic epitheliotropic intestinal T-cell lymphoma) progressed during IVE-MTX and died of lymphoma; the other is alive and disease-free. Of those with type 1 EATL, 4 are alive and lymphoma-free, 2 have relapsed from CR and 2 died during IVE-MTX.

For non-EATL patients, 1-year OS and PFS were 80\% (95\% Cl: $41-95)$ and 50\% (95\% Cl: $18-75)$, respectively. For the 13 patients (EATL and PTCL) that completed protocol treatment and received ASCT, the 1-year PFS and OS from time of ASCT were 54\% (95\% Cl: $25-76)$ and $85 \%$ (95\% Cl: $51-96)$, 
respectively. Nine deaths have been reported in total; 7 due to lymphoma, 1 treatment-related and 1 due to other causes.

This study demonstrates that IVE-MTX therapy and ASCT for frontline treatment of T-cell lymphoma can be delivered in a multi-centre setting, with a toxicity profile that is manageable and no greater than expected for this dose intense regimen and challenging patient cohort.

Although underpowered, this study provides important prospective evidence supporting use of IVEMTX and ASCT for EATL, where outcomes have historically been poor and there is a paucity of evidence to support alternative regimens. We previously reported a 5 -year PFS and OS of $52 \%$ and $60 \%$, respectively, with the same regimen in a retrospective study of 26 EATL patients ${ }^{4}$, whilst 1 -year PFS and OS were both $45 \%$ in this study. Follow-up in our study is relatively short, although relapses after ASCT tend to occur within 18 months of ASCT $^{9}$. The only other prospective trial to report outcomes for EATL patients with frontline ASCT is the Nordic Lymphoma Group NLG-T-01 study. Twenty-one EATL patients were treated with biweekly CHOP-etoposide followed by BEAM or BEAC-conditioned ASCT, with a 5-year PFS and OS of $38 \%$ and $48 \%$, respectively ${ }^{3}$. Notwithstanding the limitations of making comparisons with historical data, the results of these studies together compare favourably with a 5-year OS without ASCT of 20-28\% ${ }^{4,5}$. A retrospective European Group for Blood and Marrow Transplantation (EBMT) study of ASCT for EATL demonstrated superior outcomes for patients receiving ASCT in first remission, with a 4 -year OS was $66 \%$ compared to $36 \%$ for those in later remission ${ }^{9}$. Together, these data support use of frontline ASCT for eligible EATL patients.

Ifosfamide/etoposide-based regimens are commonly used in frontline treatment of NK/T-cell lymphoma ${ }^{10}$ and retrospective data suggest that IVE-MTX has efficacy in frontline treatment of PTCL 11. This study demonstrates that IVE-MTX has activity against PTCL but further evidence is needed to support its use as frontline therapy for PTCL subtypes other than EATL. Our results are broadly in line 
with other studies that incorporate up-front ASCT consolidation for PTCL ${ }^{12}$, including the NLG-T-01 study, which reported a 3 -year PFS and OS of $48 \%$ and $56 \%$ with CHOP-etoposide and ASCT ${ }^{3}$.

The majority of patients in this study had a PS of $0-1$, reflecting the requirement to make a judgement prior to trial registration regarding potential eligibility for ASCT. Although often impaired at diagnosis, PS may improve with successful treatment of EATL and retrospective data show that those with PS $>1$ at presentation may later become eligible for ASCT ${ }^{4}$. Fitness, PS and age may have impeded recruitment to some extent, with approximately 60 expected cases per year in the UK. However, it was also clear from discussions with UK-based haemato-oncologists that many were using IVE-MTX and ASCT for eligible patients off-trial whilst focusing research resources on trials in more common malignancies. Only 10 sites of an originally anticipated 30 recruited to this trial and funding was not available for extended recruitment. Well-designed prospective disease registries and/or international collaboration will be essential for future studies in EATL.

In summary, EATL is a rare lymphoma with extremely poor outcomes and no established standard of care. This trial provides prospective evidence, supporting previously published retrospective data ${ }^{4}$, demonstrating that IVE-MTX followed by ASCT is a feasible and effective regimen for the treatment of EATL. 


\section{Acknowledgements}

Conduct of this study was funded by Cancer Research UK (grant reference: C12372/A4755). This trial is registered at clinicaltrials.gov (NCT00669812) and EudraCT (2005-003906-27).

\section{Competing Interests}

The authors declare no relevant conflicts of interest

\section{$\underline{\text { References }}$}

$1 \quad$ Kluin-Nelemans, H. C., van Marwijk Kooy, M., Lugtenburg, P. J., van Putten, W. L., Luten, M., Oudejans, J. et al. Intensified alemtuzumab-CHOP therapy for peripheral T-cell lymphoma. Ann Oncol 22, 1595-1600, doi:10.1093/annonc/mdq635 (2011).

2 Schmitz, N., Trumper, L., Ziepert, M., Nickelsen, M., Ho, A. D., Metzner, B. et al. Treatment and prognosis of mature T-cell and NK-cell lymphoma: an analysis of patients with T-cell lymphoma treated in studies of the German High-Grade Non-Hodgkin Lymphoma Study Group. Blood 116, 3418-3425, doi:10.1182/blood-2010-02-270785 (2010).

3 d'Amore, F., Relander, T., Lauritzsen, G. F., Jantunen, E., Hagberg, H., Anderson, H. et al. Upfront autologous stem-cell transplantation in peripheral T-cell lymphoma: NLG-T-01. J Clin Oncol 30, 3093-3099, doi:10.1200/JCO.2011.40.2719 (2012).

4 Sieniawski, M., Angamuthu, N., Boyd, K., Chasty, R., Davies, J., Forsyth, P. et al. Evaluation of enteropathy-associated T-cell lymphoma comparing standard therapies with a novel regimen including autologous stem cell transplantation. Blood 115, 3664-3670, doi:10.1182/blood-2009-07-231324 (2010).

5 Delabie, J., Holte, H., Vose, J. M., Ullrich, F., Jaffe, E. S., Savage, K. J. et al. Enteropathyassociated T-cell lymphoma: clinical and histological findings from the international peripheral T-cell lymphoma project. Blood 118, 148-155, doi:10.1182/blood-2011-02-335216 (2011).

6 Gale, J., Simmonds, P. D., Mead, G. M., Sweetenham, J. W. \& Wright, D. H. Enteropathy-type intestinal T-cell lymphoma: clinical features and treatment of 31 patients in a single center. $J$ Clin Oncol 18, 795-803, doi:10.1200/JCO.2000.18.4.795 (2000).

7 Vose, J., Armitage, J., Weisenburger, D. \& International, T. C. L. P. International peripheral Tcell and natural killer/T-cell lymphoma study: pathology findings and clinical outcomes. $J$ Clin Oncol 26, 4124-4130, doi:10.1200/JCO.2008.16.4558 (2008).

8 Cheson, B. D., Pfistner, B., Juweid, M. E., Gascoyne, R. D., Specht, L., Horning, S. J. et al. Revised response criteria for malignant lymphoma. J Clin Oncol 25, 579-586, doi:10.1200/JCO.2006.09.2403 (2007).

9 Jantunen, E., Boumendil, A., Finel, H., Luan, J. J., Johnson, P., Rambaldi, A. et al. Autologous stem cell transplantation for enteropathy-associated T-cell lymphoma: a retrospective study by the EBMT. Blood 121, 2529-2532, doi:10.1182/blood-2012-11-466839 (2013).

10 Yamaguchi, M., Suzuki, R., Oguchi, M., Asano, N., Amaki, J., Akiba, T. et al. Treatments and Outcomes of Patients With Extranodal Natural Killer/T-Cell Lymphoma Diagnosed Between 2000 and 2013: A Cooperative Study in Japan. J Clin Oncol 35, 32-39, doi:10.1200/JCO.2016.68.1619 (2017). 
11 Sieniawski, M., Lennard, J., Lyons, S., Maung, Z., Mounter, P., Hervey, V. et al. Long term follow-up of patients with peripheral T-cell lymphomas treated with ifosfamide, etoposide, epirubicin, intermediate methotrexate and autologous stem cell transplantation.

Haematologica 102, Abstract P225 (2017).

12 Smith, S. M., Burns, L. J., van Besien, K., Lerademacher, J., He, W., Fenske, T. S. et al.

Hematopoietic cell transplantation for systemic mature T-cell non-Hodgkin lymphoma. J Clin Oncol 31, 3100-3109, doi:10.1200/JCO.2012.46.0188 (2013). 
Table 1: Baseline Clinical Characteristics

\begin{tabular}{lc}
\hline Baseline characteristics & $\mathrm{N}(\%)$ \\
\hline Age (years) & $\mathrm{N}=21$ \\
\hline Median (range) & $55(31$ to 71$)$ \\
\hline Sex & \\
$\quad$ Female & $7(33 \%)$ \\
\hline ECOG performance status & $11(52 \%)$ \\
O & $9(43 \%)$ \\
1 & $1(5 \%)$ \\
2 & \\
Diagnosis & $11(52 \%)$ \\
Intestinal T-cell Lymphoma/Enteropathy-type T-cell Lymphoma & $8(38 \%)$ \\
Peripheral T-cell Lymphoma, not otherwise specified & $1(5 \%)$ \\
ALK negative Anaplastic T-cell Lymphoma & $1(5 \%)$ \\
Extranodal nasal NK/T-cell Lymphoma & \\
History of coeliac disease (EATL patients only: N=11) & $3(27 \%)$ \\
Absent & $8(73 \%)$ \\
Present & \\
Lugano staging system (EATL patients only: N=11) & $5(45 \%)$ \\
I & $3(27 \%)$ \\
II-1 & $1(9 \%)$ \\
II-E & $2(18 \%)$ \\
IV & \\
Bulky disease (any tumour mass > 5cm) (EATL patients only: N=11) & $8(73 \%)$ \\
Absent & $1(9 \%)$ \\
Present & $2(18 \%)$ \\
Unknown & \\
Staging - Ann Arbor Staging Criteria (non-EATL patients: N=10) & $1(10 \%)$ \\
I & $1(10 \%)$ \\
II & $4(40 \%)$ \\
IV & $4(40 \%)$ \\
\hline
\end{tabular}


Treatment phase

CHOP (n=21) IVE/MTX ( $n=21)$

$3(14 \%)$

$13(62 \%)$

$10(77 \%)$

$17(81 \%)$

Haematological

Neutropaenia

Leukopaenia

Thrombocytopaenia

Anaemia

$\begin{array}{ll}10(48 \%) & 18(86 \%) \\ 1(5 \%) & 3(14 \%) \\ 0 & 10(48 \%) \\ 1(5 \%) & 8(38 \%)\end{array}$

$12(92 \%)$

$2(15 \%)$

21 (100\%)

$13(100 \%)$

$4(19 \%)$

$3(23 \%)$

$17(81 \%)$

$11(52 \%)$

\section{Gastrointestinal}

Diarrhoea

Gl perforation

Nausea and vomiting

GI haemorrhage

Other GI

0

$1(5 \%)$

0

0

$2(10 \%)$

$1(5 \%)$

$1(5 \%)$

0

$2(10 \%)$

0

$5(38 \%)$

$5(24 \%)$

0

$1(8 \%)$

$3(14 \%)$

$2(10 \%)$

0

$1(5 \%)$

\section{Other systems}

\section{Cardiac}

$1(5 \%)$

$1(5 \%)$

$1(5 \%)$

$1(5 \%)$
0

0

Encephalopathy

Renal dysfunction

Pulmonary

Hypokalaemia

0
Raised ALT
2 (9\%)

0

1 (5\%)

3 (14\%)

2 (10\%)

$1(5 \%)$

$1(5 \%)$
$2(10 \%)$

0

0

$1(8 \%)$

$1(8 \%)$

$2(9 \%)$

$2(10 \%)$

$3(14 \%)$

$1(8 \%)$

$4(19 \%)$

$2(10 \%)$

$1(5 \%)$

$2(10 \%)$

Table 2: Grade 3-5 adverse events according to treatment phase (all patients)

Includes all events occurring in 2 or more patients, and all gastrointestinal toxicity. ALT= alanine transaminase, $\mathrm{Gl}=$ gastrointestinal. Toxicity was assessed according to the National Cancer Institute Common Terminology Criteria for Adverse Events version 3.0. 
Figure 1: Patient outcomes- overall survival (A) and progression-free survival (B) for the whole cohort, and overall survival according to diagnosis (C)

A)

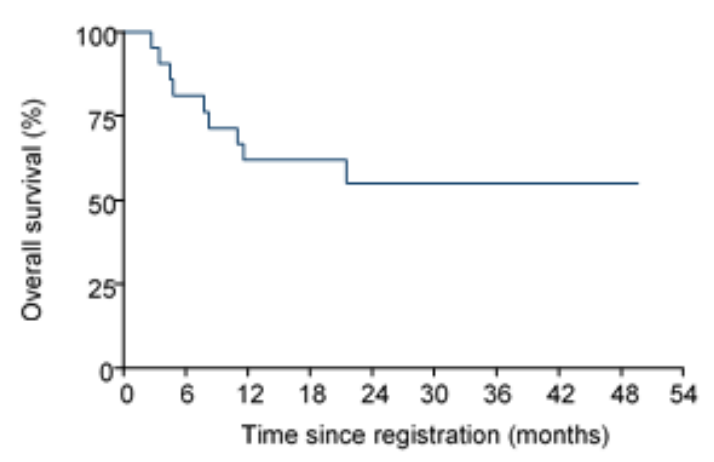

$\begin{array}{lllllllllll}\text { Number at risk: } & 21 & 17 & 13 & 11 & 7 & 4 & 3 & 1 & 1 & 0\end{array}$

B)

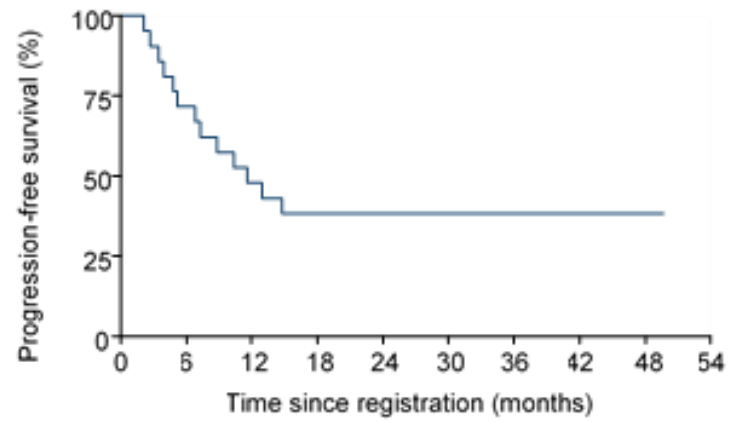

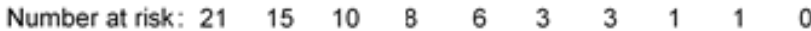

C)

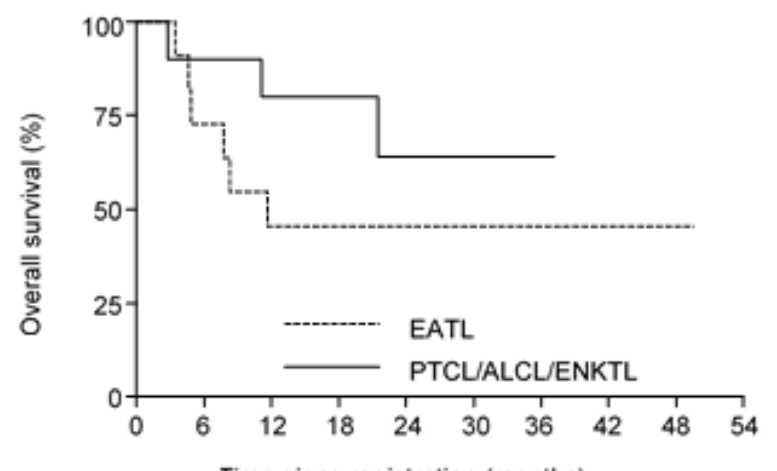

Number at risk

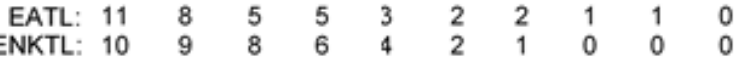

